\title{
LANDEN INEQUALITIES FOR SPECIAL FUNCTIONS
}

\author{
ÁRPÁD BARICZ \\ (Communicated by Walter Van Assche)
}

\begin{abstract}
In this paper our aim is to present some Landen inequalities for Gaussian hypergeometric functions, confluent hypergeometric functions, generalized Bessel functions and general power series. Our main results complement and generalize some known results in the literature.
\end{abstract}

\section{INTRODUCTION}

Let us consider the Gaussian hypergeometric function $F(a, b ; c ; \cdot):(-1,1) \rightarrow \mathbb{R}$, which for real numbers $a, b$ and $c$ such that $c$ is not in $\{0,-1, \ldots\}$ has the infinite series representation

$$
F(a, b ; c ; x):={ }_{2} F_{1}(a, b ; c ; x)=\sum_{n \geq 0} \frac{(a)_{n}(b)_{n}}{(c)_{n}} \cdot \frac{x^{n}}{n !},
$$

where $x \in(-1,1),(a)_{0}=1$ for $a \neq 0$ and $(a)_{n}=a(a+1) \ldots(a+n-1)=$ $\Gamma(a+n) / \Gamma(a)$ for each $n \in\{1,2, \ldots\}$ denotes the Pochhammer (or Appell) symbol. Some of the most important properties of the complete elliptic integral of the first kind, i.e. $\mathcal{K}(r)$, defined by

$$
\mathcal{K}(r)=\frac{\pi}{2} F\left(\frac{1}{2}, \frac{1}{2} ; 1 ; r^{2}\right)=\int_{0}^{\frac{\pi}{2}}\left(1-r^{2} \sin ^{2} t\right)^{-\frac{1}{2}} d t, \quad r \in(0,1),
$$

are the Landen identities proved in 1771, [1]:

$$
\mathcal{K}\left(\frac{2 \sqrt{r}}{1+r}\right)=(1+r) \mathcal{K}(r), \quad \mathcal{K}\left(\frac{1-r}{1+r}\right)=\frac{1+r}{2} \mathcal{K}\left(\sqrt{1-r^{2}}\right) .
$$

These Landen identities, which are in fact equivalent to each other, have been the starting points of the investigations of Qiu and Vuorinen 13, and recently of Simić and Vuorinen [14. In this paper, motivated by [14, we make a contribution to the subject by showing that [14, Theorem 2.1], proved for the zero-balanced hypergeometric function $F(a, b ; a+b ; \cdot)$, can be extended to the hypergeometric function $F(a, b ; c ; \cdot)$ and also to general power series. Moreover, we prove that, by using a generalization of the first Landen identity in (11), the Landen inequalities for the Gaussian hypergeometric functions can be improved in some cases. Our main results complement the results from [6, 8, 13] and [14].

Received by the editors August 23, 2012 and, in revised form, September 10, 2012.

2010 Mathematics Subject Classification. Primary 39B62, 33C05.

Key words and phrases. Hypergeometric functions, power series, Landen inequalities. 


\section{LANDEN INEQUALITIES FOR POWER SERIES}

Let us recall a result of Biernacki and Krzyż 9], which we will use in the sequel.

Lemma 1. Consider the power series $f(x)=\sum_{n \geq 0} a_{n} x^{n}$ and $g(x)=\sum_{n \geq 0} b_{n} x^{n}$, where $a_{n} \in \mathbb{R}$ and $b_{n}>0$ for all $n \in\{0,1, \ldots\}$, and suppose that both converge on $(-r, r)$, $r>0$. If the sequence $\left\{a_{n} / b_{n}\right\}_{n \geq 0}$ is increasing (decreasing), then the function $x \mapsto f(x) / g(x)$ is increasing (decreasing) too on $(0, r)$.

For different proofs and various applications of this result the interested reader is referred to the papers $[2,3,5,8,10,12,14]$ and to the references therein.

Our first main result is the following theorem.

Theorem 1. Let $a, b, c \in \mathbb{R}$ such that $c$ is not a negative integer or zero and consider the function $Q:(0,1) \rightarrow(0, \infty)$, defined by $Q(x)=F(a, b ; c ; x) / F\left(\frac{1}{2}, \frac{1}{2} ; 1 ; x\right)$. The following assertions are true:

a. If $a+b \geq c$ and $4 a b \geq \max \{1, c\}$, then $Q$ is increasing, and consequently

$$
\begin{gathered}
F\left(a, b ; c ; \frac{4 r}{(1+r)^{2}}\right) \geq(1+r) \cdot F\left(a, b ; c ; r^{2}\right), \\
F\left(a, b ; c ;\left(\frac{1-r}{1+r}\right)^{2}\right) \leq \frac{1+r}{2} \cdot F\left(a, b ; c ; 1-r^{2}\right)
\end{gathered}
$$

hold for each $r \in(0,1)$.

b. If $a+b \leq c$ and $4 a b \leq \min \{1, c\}$, then $Q$ is decreasing, and consequently

$$
\begin{gathered}
F\left(a, b ; c ; \frac{4 r}{(1+r)^{2}}\right) \leq(1+r) \cdot F\left(a, b ; c ; r^{2}\right), \\
F\left(a, b ; c ;\left(\frac{1-r}{1+r}\right)^{2}\right) \geq \frac{1+r}{2} \cdot F\left(a, b ; c ; 1-r^{2}\right)
\end{gathered}
$$

hold for each $r \in(0,1)$.

Proof of $\mathbf{a}$ and $\mathbf{b}$. We shall apply Lemma 1. Since $Q(x)$ can be rewritten as

$$
Q(x)=\frac{F(a, b ; c ; x)}{F\left(\frac{1}{2}, \frac{1}{2} ; 1 ; x\right)}=\frac{\sum_{n \geq 0} \frac{(a)_{n}(b)_{n}}{(c)_{n}} \cdot \frac{x^{n}}{n !}}{\sum_{n \geq 0} \frac{\left(\frac{1}{2}\right)_{n}\left(\frac{1}{2}\right)_{n}}{(1)_{n}} \cdot \frac{x^{n}}{n !},}
$$

in view of Lemma 1, the monotonicity of the quotient $Q$ depends on the monotonicity of the quotient sequence $\left\{\alpha_{n}\right\}_{n \geq 0}$, defined by

$$
\alpha_{n}=\frac{(a)_{n}(b)_{n}}{(c)_{n}} \cdot \frac{(1)_{n}}{\left(\frac{1}{2}\right)_{n}\left(\frac{1}{2}\right)_{n}} .
$$

Now, observe that

$$
\frac{\alpha_{n+1}}{\alpha_{n}}=\frac{(n+a)(n+b)(n+1)}{(n+c)\left(n+\frac{1}{2}\right)\left(n+\frac{1}{2}\right)} \geq 1
$$


if and only if

$$
\Delta_{n}=(a+b-c) n^{2}+\left(a+b-c+a b-\frac{1}{4}\right) n+a b-\frac{c}{4} \geq 0 .
$$

Thus, if $a+b \geq c$ and $4 a b \geq \max \{1, c\}$, then $\Delta_{n} \geq 0$ for all $n \in\{0,1, \ldots\}$; that is, the sequence $\left\{\alpha_{n}\right\}_{n \geq 0}$ is increasing, and consequently by using Lemma 1 the function $Q$ is increasing. In other words, if $0<x<y<1$, then we have $Q(x)<Q(y)$. Now, choosing $x=x(r)=r^{2}$ and $y=y(r)=4 r /(1+r)^{2}$, we obtain the inequality

$$
\frac{F\left(a, b ; c ; r^{2}\right)}{F\left(\frac{1}{2}, \frac{1}{2} ; 1 ; r^{2}\right)} \leq \frac{F\left(a, b ; c ; \frac{4 r}{(1+r)^{2}}\right)}{F\left(\frac{1}{2}, \frac{1}{2} ; 1 ; \frac{4 r}{(1+r)^{2}}\right)}
$$

that is,

$$
F\left(a, b ; c ; r^{2}\right) \leq F\left(a, b ; c ; \frac{4 r}{(1+r)^{2}}\right) \cdot \frac{\mathcal{K}(r)}{\mathcal{K}\left(\frac{2 \sqrt{r}}{1+r}\right)},
$$

which in view of the first Landen identity in (11) is equivalent to (2). Similarly, by choosing $x=x(r)=[(1-r) /(1+r)]^{2}$ and $y=y(r)=1-r^{2}$ we get the inequality

$$
\frac{F\left(a, b ; c ;\left(\frac{1-r}{1+r}\right)^{2}\right)}{F\left(\frac{1}{2}, \frac{1}{2} ; 1 ;\left(\frac{1-r}{1+r}\right)^{2}\right)} \leq \frac{F\left(a, b ; c ; 1-r^{2}\right)}{F\left(\frac{1}{2}, \frac{1}{2} ; 1 ; 1-r^{2}\right)},
$$

that is,

$$
F\left(a, b ; c ;\left(\frac{1-r}{1+r}\right)^{2}\right) \leq F\left(a, b ; c ; 1-r^{2}\right) \cdot \frac{\mathcal{K}\left(\frac{1-r}{1+r}\right)}{\mathcal{K}\left(\sqrt{1-r^{2}}\right)},
$$

which in view of the second Landen identity in (1) is equivalent to (3). This proves part $\mathbf{a}$. The proof of part $\mathbf{b}$ is similar, and thus we omit the details.

First of all we mention that the Landen inequalities (2) and (3) are equivalent, as well as the inequalities (44) and (5). Namely, if we change $r$ to $(1-r) /(1+r)$ in (2) and (4), then we obtain (3) and (5). Similarly, if we change $(1-r) /(1+r)$ to $r$ in (3) and (5), then we obtain (2) and (4). It should also be mentioned here that in Theorem 1 it is not necessary to assume that $a, b$ and $c$ are positive numbers. However, if we suppose in inequalities (2) and (4), in particular that $a, b>0$ and $c=a+b$, then we reobtain [14, Theorem 2.1], which was obtained recently by Simić and Vuorinen. We mention that the condition $4 a b \geq \max \{1, c\}$ in part a of Theorem 1 reduces to $4 a b \geq a+b$, since by applying the arithmetic mean - geometric mean inequality for the numbers $a$ and $b$, the above condition implies that $4 a b \geq 1$. Similarly, the condition $4 a b \leq \min \{1, c\}$ in part $\mathbf{b}$ of Theorem 1 reduces to $4 a b \leq 1$, since by applying the geometric mean - harmonic mean inequality for the numbers $a$ and $b$, the above condition implies that $4 a b \leq a+b$. We also note that a general result about the monotonicity of quotients of Gaussian hypergeometric functions was given by Ponnusamy and Vuorinen in [12, Theorem 2.31]. Finally, we note that the inequality (5) was proved earlier by Qiu and Vuorinen [13, Theorem 1.2], but just for $a, b \in(0,1)$ and $c=a+b \leq 1$. Observe that in this case the condition $4 a b \leq 1$ is clearly satisfied since $2 \sqrt{a b} \leq a+b \leq 1$. 
Now, let us consider the sequence $\left\{\omega_{n}\right\}_{n \geq 0}$, defined by

$$
\omega_{n}=\left[\frac{(1)_{n}}{\left(\frac{1}{2}\right)_{n}}\right]^{2}=\pi \cdot\left[\frac{\Gamma(n+1)}{\Gamma\left(n+\frac{1}{2}\right)}\right]^{2} .
$$

By using this sequence we would like to show a generalization of Theorem 1 . Note that since the proof of this general result proceeds along the lines introduced in the proof of Theorem 1, we omit the details. This result complements [6, Theorem 3.1].

Theorem 2. Suppose that the power series $f(x)=\sum_{n \geq 0} a_{n} x^{n}$ is convergent for all $x \in(0,1)$, where $a_{n} \in \mathbb{R}$ for all $n \in\{0,1, \ldots\}$, and assume that the sequence $\left\{a_{n} \cdot \omega_{n}\right\}_{n \geq 0}$ is increasing. Then the function $x \mapsto f(x) / \mathcal{K}(\sqrt{x})$ is increasing on $(0,1)$, and by using the notation $\lambda_{f}(x)=f\left(x^{2}\right)$ we have the Landen type inequality for all $r \in(0,1)$ :

$$
\lambda_{f}\left(\frac{2 \sqrt{r}}{1+r}\right) \geq(1+r) \cdot \lambda_{f}(r) .
$$

Moreover, if the sequence $\left\{a_{n} \cdot \omega_{n}\right\}_{n \geq 0}$ is decreasing, then $x \mapsto f(x) / \mathcal{K}(\sqrt{x})$ is decreasing on $(0,1)$, and consequently (6) is reversed.

Observe that the sequence $\{\omega\}_{n \geq 0}$ is increasing. Thus if the sequence $\left\{a_{n}\right\}_{n \geq 0}$ is also increasing, then the power series $f(x)$ of Theorem 2 immediately satisfies the Landen type inequality (6), which is in fact equivalent to

$$
\lambda_{f}\left(\frac{1-r}{1+r}\right) \leq \frac{1+r}{2} \cdot \lambda_{f}\left(\sqrt{1-r^{2}}\right) .
$$

Note that if we consider, as in [6,, , the generalized Bessel function $u_{\nu}:(0, \infty) \rightarrow \mathbb{R}$ and the Kummer hypergeometric function $\Phi(p, q ; \cdot):(0, \infty) \rightarrow \mathbb{R}$, defined by

$$
u_{\nu}(x)=\sum_{n \geq 0} \frac{\left(-\frac{c}{4}\right)^{n}}{(\kappa)_{n}} \cdot \frac{x^{n}}{n !} \text { and } \Phi(p, q ; x)=\sum_{n \geq 0} \frac{(p)_{n}}{(q)_{n}} \cdot \frac{x^{n}}{n !}
$$

where $\nu, b, c, p, q \in \mathbb{R}, \kappa=\nu+\frac{b+1}{2}$ and $q$ are not in $\{0,-1, \ldots\}$, then it can be shown that the sequences

$$
\left\{\frac{\left(-\frac{c}{4}\right)^{n}}{(\kappa)_{n} n !} \cdot \omega_{n}\right\}_{n \geq 0} \text { and }\left\{\frac{(p)_{n}}{(q)_{n} n !} \cdot \omega_{n}\right\}_{n \geq 0}
$$

are decreasing if $\kappa \geq \max \left\{0,-c,-\frac{c+1}{4}\right\}$ and $q \geq \max \left\{0,4 p, p+\frac{3}{4}\right\}$. Thus, if use the notation $\lambda_{\nu}(r)=u_{\nu}\left(r^{2}\right)$ and $\lambda_{\Phi}(r)=\Phi\left(p, q ; r^{2}\right)$, then in view of Theorem 4 we obtain the following result. Note that this result complements [6, Theorem 2.3] and [6, Corollary 3.2].

Theorem 3. Let $\nu, b, c, p$ and $q$ be real numbers such that $\kappa \geq \max \left\{-1,-c,-\frac{c+1}{4}\right\}$ and $q \geq \max \left\{0,4 p, p+\frac{3}{4}\right\}$. Then $x \mapsto u_{\nu}(x) / \mathcal{K}(\sqrt{x})$ and $x \mapsto \Phi(p, q ; x) / \mathcal{K}(\sqrt{x})$ are decreasing on $(0,1)$, and consequently for all $r \in(0,1)$ we have

$$
\lambda_{\nu}\left(\frac{2 \sqrt{r}}{1+r}\right) \leq(1+r) \cdot \lambda_{\nu}(r) \text { and } \lambda_{\Phi}\left(\frac{2 \sqrt{r}}{1+r}\right) \leq(1+r) \cdot \lambda_{\Phi}(r) .
$$


Now, let us consider the following hypergeometric transformation [4, p. 128]:

$$
F\left(a, b ; 2 b ; \frac{4 r}{(1+r)^{2}}\right)=(1+r)^{2 a} \cdot F\left(a, a+\frac{1}{2}-b ; b+\frac{1}{2} ; r^{2}\right),
$$

which can be regarded as the generalization of the first Landen identity in (11). By using this transformation we can obtain the following result. Observe that if the conditions of part a of Theorem 4 are valid and in addition $c>0$ and $a \geq \frac{1}{2}$, then the Landen inequality (8) improves (2). Similarly, if the conditions of part b of Theorem 4 are valid and in addition $a \leq \frac{1}{2}$, then the Landen inequality (9) improves (4).

Theorem 4. Let $a, b>0$ and $c \in \mathbb{R}$ such that $c$ is not a negative integer or zero. The following assertions are true:

a. If $\max \{1, c\} \leq 2 b \leq a+\frac{1}{2}$ or $c \leq 2 b \leq a$ or $3 c \leq 6 b \leq \min \{6 a, 4 a+1\}$, then

$$
F\left(a, b ; c ; \frac{4 r}{(1+r)^{2}}\right) \geq(1+r)^{2 a} \cdot F\left(a, b ; c ; r^{2}\right)
$$

holds for each $r \in(0,1)$.

b. If $a+\frac{1}{2} \leq 2 b \leq \min \{1, c\}$ or $\max \{6 a, 4 a+1\} \leq 6 b \leq 3 c$, then

$$
F\left(a, b ; c ; \frac{4 r}{(1+r)^{2}}\right) \leq(1+r)^{2 a} \cdot F\left(a, b ; c ; r^{2}\right)
$$

holds for each $r \in(0,1)$.

Proof of $\mathbf{a}$ and $\mathbf{b}$. We proceed similarly as in the proof of Theorem 1 For this, first we consider the function $T:(0,1) \rightarrow(0, \infty)$, defined by

$$
T(x)=\frac{F(a, b ; c ; x)}{F(a, b ; 2 b ; x)}=\frac{\sum_{n \geq 0} \frac{(a)_{n}(b)_{n}}{(c)_{n}} \cdot \frac{x^{n}}{n !}}{\sum_{n \geq 0} \frac{(a)_{n}(b)_{n}}{(2 b)_{n}} \cdot \frac{x^{n}}{n !}} .
$$

Now, in view of Lemma1, for the monotonicity of the quotient $T$ we need to study the monotonicity of the quotient sequence $\left\{\beta_{n}\right\}_{n \geq 0}$, defined by $\beta_{n}=(2 b)_{n} /(c)_{n}$. Since $\beta_{n+1} / \beta_{n}=(n+2 b) /(n+c)$, it is clear that the sequence $\left\{\beta_{n}\right\}_{n \geq 0}$ is increasing (decreasing) if $2 b \geq c(2 b \leq c)$. Now, if we consider the case $2 b \geq c$, then $\left\{\beta_{n}\right\}_{n \geq 0}$ is increasing, and applying Lemma 1 the function $T$ is increasing. In other words, if $0<x<y<1$, then we have $T(x)<T(y)$. Thus, choosing $x=x(r)=r^{2}$ and $y=y(r)=4 r /(1+r)^{2}$, we obtain the inequality

$$
\frac{F\left(a, b ; c ; r^{2}\right)}{F\left(a, b ; 2 b ; r^{2}\right)} \leq \frac{F\left(a, b ; c ; \frac{4 r}{(1+r)^{2}}\right)}{F\left(a, b ; 2 b ; \frac{4 r}{(1+r)^{2}}\right)},
$$

which in view of (7) is equivalent to

$$
F\left(a, b ; c ; r^{2}\right) \leq F\left(a, b ; c ; \frac{4 r}{(1+r)^{2}}\right) \cdot \frac{F\left(a, b ; 2 b ; r^{2}\right)}{(1+r)^{2 a} F\left(a, a+\frac{1}{2}-b ; b+\frac{1}{2} ; r^{2}\right)} .
$$

Observe that if $2 b \leq a+\frac{1}{2}$ and $2 b \geq 1$, then for all $n \in\{0,1, \ldots\}$ we have $(b)_{n} \leq$ $\left(a+\frac{1}{2}-b\right)_{n}$ and $\left(b+\frac{1}{2}\right)_{n} \leq(2 b)_{n}$. Similarly, if $0<2 b \leq a$, then we clearly have 
$\left(b+\frac{1}{2}\right)_{n} \leq\left(a+\frac{1}{2}-b\right)_{n}$ and $(b)_{n}<(2 b)_{n}$ for all $n \in\{0,1, \ldots\}$. In both cases we have

$$
\frac{(b)_{n}}{(2 b)_{n}} \cdot \frac{(a)_{n}}{n !} \leq \frac{\left(a+\frac{1}{2}-b\right)_{n}}{\left(b+\frac{1}{2}\right)_{n}} \cdot \frac{(a)_{n}}{n !}
$$

for all $n \in\{0,1, \ldots\}$, and consequently for all $r \in(0,1)$ one has

$$
F\left(a, b ; 2 b ; r^{2}\right) \leq F\left(a, a+\frac{1}{2}-b ; b+\frac{1}{2} ; r^{2}\right) .
$$

On the other hand, observe that if $a \geq b$ and $2 a+\frac{1}{2} \geq 3 b$, then for all $n \in\{0,1, \ldots\}$ we have $(a-b) n+b\left(2 a+\frac{1}{2}-3 b\right) \geq 0$, and consequently the sequence $\left\{\gamma_{n}\right\}_{n \geq 0}$, defined by

satisfies

$$
\gamma_{n}=\frac{(b)_{n}\left(b+\frac{1}{2}\right)_{n}}{(2 b)_{n}\left(a+\frac{1}{2}-b\right)_{n}},
$$

$$
\frac{\gamma_{n+1}}{\gamma_{n}}=\frac{(n+b)\left(n+b+\frac{1}{2}\right)}{(n+2 b)\left(n+a+\frac{1}{2}-b\right)} \leq 1
$$

for all $n \in\{0,1, \ldots\}$. Thus, by using Lemma 1, the function

$$
r \mapsto \frac{F(a, b ; 2 b ; r)}{F\left(a, a+\frac{1}{2}-b ; b+\frac{1}{2} ; r\right)}
$$

is decreasing on $(0,1)$, and consequently

$$
\frac{F(a, b ; 2 b ; r)}{F\left(a, a+\frac{1}{2}-b ; b+\frac{1}{2} ; r\right)}<\lim _{r \searrow 0} \frac{F(a, b ; 2 b ; r)}{F\left(a, a+\frac{1}{2}-b ; b+\frac{1}{2} ; r\right)}=1
$$

for all $r \in(0,1)$. Now, changing $r$ to $r^{2}$ we again obtain (11), and combining (10) with (11) we obtain (8). This proves part a. The proof of part $\mathbf{b}$ is similar, and thus we omit the details.

We mention that by changing $r$ to $(1-r) /(1+r)$ in inequality (8) we obtain for all $r \in(0,1)$ the Landen inequality

$$
F\left(a, b ; c ;\left(\frac{1-r}{1+r}\right)^{2}\right) \leq\left(\frac{1+r}{2}\right)^{2 a} \cdot F\left(a, b ; c ; 1-r^{2}\right),
$$

where $a, b$ and $c$ are as in part a of Theorem 4. Moreover, if $a, b$ and $c$ are as in part $\mathbf{b}$ of Theorem 4 , then the above Landen inequality is reversed. Note that these inequalities can also be obtained by using the steps of the proof of Theorem 4 and the formula

$$
F\left(a, b ; 2 b ; 1-r^{2}\right)=\left(\frac{1+r}{2}\right)^{-2 a} \cdot F\left(a, a+\frac{1}{2}-b ; b+\frac{1}{2} ;\left(\frac{1-r}{1+r}\right)^{2}\right),
$$

which is the generalization of the second Landen identity in (11) and readily follows from (7) by changing $r$ to $(1-r) /(1+r)$, or from [4, p. 132]

$$
F\left(a, b ; 2 b ; r^{2}\right)=\left(\frac{1+\sqrt{1-r^{2}}}{2}\right)^{-2 a} \cdot F\left(a, a+\frac{1}{2}-b ; b+\frac{1}{2} ;\left(\frac{1-\sqrt{1-r^{2}}}{1+\sqrt{1-r^{2}}}\right)^{2}\right)
$$

by replacing $r$ with $\sqrt{1-r^{2}}$. 
Finally, we note that for some rational values of $(a, b, c)$ the hypergeometric function $F(a, b ; c ; \cdot)$ reduces to some well-known special elementary functions, and thus the results of Theorems 1 and 4 yield Landen inequalities for many elementary functions. For a list of elementary representations we refer to [11, pp. 386-387] and the references therein. For example, if we choose the triplets $(a, b, c)=\left(\frac{1}{2}, \frac{1}{2}, \frac{3}{2}\right)$ and $(a, b, c)=\left(\frac{1}{2}, 1, \frac{3}{2}\right)$, then in view of the representations

$$
\begin{gathered}
F\left(\frac{1}{2}, \frac{1}{2} ; \frac{3}{2} ; r^{2}\right)=\frac{1}{r} \arcsin r, \\
F\left(\frac{1}{2}, 1 ; \frac{3}{2} ; r^{2}\right)=\frac{1}{2 r} \log \left(\frac{1+r}{1-r}\right),
\end{gathered}
$$

and the inequalities (2) and (9), we obtain the next Landen inequalities for $r \in$ $(0,1)$ :

$$
\begin{gathered}
\frac{\sqrt{r}}{2} \arcsin \left(\frac{2 \sqrt{r}}{1+r}\right)<\arcsin r \\
\left(\frac{1+\sqrt{r}}{1-\sqrt{r}}\right)^{\sqrt{r}}>\frac{1+r}{1-r}
\end{gathered}
$$

\section{ACKNOWLEDGMENTS}

This work was supported by the János Bolyai Scholarship of the Hungarian Academy of Sciences. The author wishes to acknowledge the referee's comments and suggestions, which enhanced this paper.

\section{REFERENCES}

[1] Gert Almkvist and Bruce Berndt, Gauss, Landen, Ramanujan, the arithmetic-geometric mean, ellipses, $\pi$, and the Ladies diary, Amer. Math. Monthly 95 (1988), no. 7, 585-608, DOI 10.2307/2323302. MR966232 (89j:01028)

[2] Horst Alzer and Song-Liang Qiu, Monotonicity theorems and inequalities for the complete elliptic integrals, J. Comput. Appl. Math. 172 (2004), no. 2, 289-312, DOI 10.1016/j.cam.2004.02.009. MR2095322(2005i:33021)

[3] G. D. Anderson, M. K. Vamanamurthy, and M. Vuorinen, Generalized convexity and inequalities, J. Math. Anal. Appl. 335 (2007), no. 2, 1294-1308, DOI 10.1016/j.jmaa.2007.02.016. MR2346906 (2008i:33012)

[4] George E. Andrews, Richard Askey, and Ranjan Roy, Special functions, Encyclopedia of Mathematics and its Applications, vol. 71, Cambridge University Press, Cambridge, 1999. MR.1688958 (2000g:33001)

[5] R. Balasubramanian, S. Ponnusamy, and M. Vuorinen, Functional inequalities for the quotients of hypergeometric functions, J. Math. Anal. Appl. 218 (1998), no. 1, 256-268, DOI 10.1006/jmaa.1997.5776. MR 1601889 (99k:33007)

[6] Árpád Baricz, Landen-type inequality for Bessel functions, Comput. Methods Funct. Theory 5 (2005), no. 2, 373-379, DOI 10.1007/BF03321104. MR2205420 (2008i:33019)

[7] Árpád Baricz, Bounds for modified Bessel functions of the first and second kinds, Proc. Edinb. Math. Soc. (2) 53 (2010), no. 3, 575-599, DOI 10.1017/S0013091508001016. MR2720238 (2011k:33010)

[8] Árpád Baricz, Generalized Bessel functions of the first kind, Lecture Notes in Mathematics, vol. 1994, Springer-Verlag, Berlin, 2010. MR2656410 (2011f:33007)

[9] Mieczysław Biernacki and Jan Krzyż, On the monotonity of certain functionals in the theory of analytic functions (English, with Polish and Russian summaries), Ann. Univ. Mariae Curie-Skłodowska. Sect. A. 9 (1955), 135-147 (1957). MR0089903 (19,736f) 
[10] Ville Heikkala, Mavina K. Vamanamurthy, and Matti Vuorinen, Generalized elliptic integrals, Comput. Methods Funct. Theory 9 (2009), no. 1, 75-109, DOI 10.1007/BF03321716. MR.2478265 (2009k:33016)

[11] NIST handbook of mathematical functions, U.S. Department of Commerce, National Institute of Standards and Technology, Washington, DC, 2010. Edited by Frank W. J. Olver, Daniel W. Lozier, Ronald F. Boisvert and Charles W. Clark; with 1 CD-ROM (Windows, Macintosh and UNIX). MR2723248 (2012a:33001)

[12] S. Ponnusamy and M. Vuorinen, Asymptotic expansions and inequalities for hypergeometric functions, Mathematika 44 (1997), no. 2, 278-301, DOI 10.1112/S0025579300012602. MR 1600537 (99b:33004)

[13] S.-L. Qiu and M. Vuorinen, Landen inequalities for hypergeometric functions, Nagoya Math. J. 154 (1999), 31-56. MR.1689171 (2001g:33004)

[14] Slavko Simić and Matti Vuorinen, Landen inequalities for zero-balanced hypergeometric functions, Abstr. Appl. Anal. (2012), Art. ID 932061, 11. MR2898059

Department of Economics, Babeş-Bolyai University, Cluj-Napoca 400591, Romania

E-mail address: bariczocsi@yahoo.com 